\title{
Temperature changes in the function of the shape inherent to the band of exciton absorption in nanofilm of layered semiconductor
}

\author{
A.V. Derevyanchuk, O.V. Pugantseva, V.M. Kramar \\ Yuriy Fedkovych Chernivtsi National University, \\ 2, Kotsyubynsky str., 58012 Chernivtsi, Ukraine; \\ E-mail: v.kramar@chnu.edu.ua
}

\begin{abstract}
Represented in this paper is the method and results of theoretical investigations aimed at the influence of spatial confinement effects, self-polarization of heterojunction planes as well as exciton-phonon interaction on the position and shape of the band corresponding to exciton absorption in nanofilms of layered semiconductor in a dielectric matrix. The heterojunction is considered as unloaded, the nanosystem is modeled by an infinitely deep quantum well and characterized by an essential difference between dielectric permittivities on both sides of the heterojunction. Calculated in this work are the dependences for the form-function of the absorption band on the thickness of lead iodide nanofilm embedded into polymer E-MAA or glass, and on its temperature. The results of calculations are in good accordance with known data of experimental measurements.
\end{abstract}

Keywords: nanoheterostructure, quantum well, nanofilm, exciton, absorption, layered semiconductor, lead iodide.

Manuscript received 23.01.14; revised version received 29.04.14; accepted for publication 12.06.14; published online 30.06.14.

\section{Introduction}

Intense development of technologies to obtain and study physical properties of low-dimensional heterostructures based on lead iodide is related with the possibility to use them for creation of X-ray and $\gamma$-radiation detectors as well as other optoelectronic devices [1-9]. Spatial confinement of quasi-particles in low-dimensional structures cause additional quantization of their motion characteristics, which is pronounced in experimentally observed changes in their optical properties, including those in the excitonic range of the spectrum [3-6].

Theoretical investigations of exciton states in nanostructures with single quantum wells (QW) were performed by many authors with various methods and approaches (see, for instance, [10-13]), however, the nanostructures based on layered crystals were not considered in them.

Adduced in [14] are the results of theoretical investigations devoted to temperature changes in the energy of transition to the ground exciton state in a plain nano-film (NF) of $2 \mathrm{H}$-polytype of lead iodide $\left(2 \mathrm{H}-\mathrm{PbI}_{2}\right)$ embedded into dielectric surroundings - borosilicate glass or ethylene-methacryl acid (E-MAA) copolymer. Availability of the method to calculate the exciton energy in NF enables to study temperature transformations in the shape of the exciton absorption band in this nanosystem. The method and results of this investigation are enlightened in this paper below.

\section{Choosing the model of investigations and setting the task}

Investigation of the character of temperature changes in the bands corresponding to exciton transitions in semiconductor crystals can be performed using the model of Wannier-Mott exciton created as a result of the direct electron phototransition. The spectral dependence of the absorption coefficient related with these transitions can be expressed using the following function

$$
\begin{aligned}
& \alpha(\omega, T)=\frac{2 \pi\left|D_{0}(\omega)\right|^{2} \Gamma(\omega, T)}{|\hbar \omega-E-\Delta(\omega, T)|^{2}+\Gamma^{2}(\omega, T)}= \\
& =2 \pi\left|D_{0}(\omega)\right|^{2} S(\omega, T)
\end{aligned}
$$

that depends on temperature as a parameter. Here,

$$
S(\omega, T)=\frac{\Gamma(\omega, T)}{|\hbar \omega-E-\Delta(\omega, T)|^{2}+\Gamma^{2}(\omega, T)}
$$


is the function of the shape inherent to the exciton absorption band related with the transition to the exciton state; $D_{0}$ and $E$ are matrix element of the electric dipole moment and the energy of this transition, respectively; $\hbar \omega$ is the energy of exciting wave; $\Delta(\omega, T)$ and $\Gamma(\omega, T)$ are real and imaginary parts of the mass operator

$M(\omega, T)=\Delta(\omega, T)-i \Gamma(\omega, T)$

for the Green function of the exciton-phonon system

$$
G(\omega, T)=\frac{1}{\hbar \omega-E+M(\omega, T)}
$$

The model for the Wannier-Mott exciton in NF of layered semiconductor (medium 0) embedded into isotropic dielectric (medium 1) was considered in [14]. The heterojunction is considered here as unstrained, while QW is rectangular and, with account of dielectric nature of barrier medium, the infinitely deep one.

If the origin of the Cartesian coordinate system is placed into the NF center in such a manner that XOY plane is parallel to its surface, and $\mathrm{OZ}$ axis coincides with the crystallographic axis $\mathrm{C}$ of the layered crystal, then the NF thickness (i.e., the QW width) can be considered as equal to $a=N c$, where $c$ is the lattice parameter along $\mathrm{C}$-axis, while $N$ is the natural number equal to the amount of $\mathrm{PbI}_{2}$ layers in NF well material (medium 0).

The Hamiltonian for this exciton-phonon system necessary to find the mass operator (3) should be constructed using the approach of effective masses for the electron sub-system and in the form of dielectric continuum - for the phonon one [15], and takes the following look:

$\hat{H}=\hat{H}_{e x}+\hat{H}_{p h}+\hat{H}_{\text {int }} \cdot$

The operators $\hat{H}_{e x}$ and $\hat{H}_{p h}$ describe, respectively, the state of free electron and free phonon, while $\hat{H}_{\text {int }}$ - exciton-phonon interaction (EPI); their apparent look and peculiarities in the case of NF consisting of layered semiconductor are described in detail in $[14,16]$, while offered in [13] is the method for finding the energy of quasi-two-dimensional exciton in $\mathrm{NF}$ as well as the function of its state. In the first approximation of the perturbulation theory, the exciton wave-function can be represented in the form of production

$$
\Psi_{v}\left(\vec{\rho}, z_{e}, z_{h}\right)=\psi_{n_{e}}\left(z_{e}\right) \psi_{n_{h}}\left(z_{h}\right) \varphi_{n m}(\vec{\rho})
$$

of functions $\psi_{n_{p}}\left(z_{p}\right)$ that describe quantized states of non-interacting electron $(p=e)$ and hole $(p=h)$, which corresponds to their motion along the direction of the film growth, as well as the function $\varphi_{n m}(\vec{\rho})$ of their state bound by Coulomb interaction - their relative motion in the plane of heterojunction $\left(\vec{\rho}=\left(x_{e}-x_{h}, y_{e}-y_{h}\right)\right)$.
Here, $v=\left(n_{e}, n_{h}, n, m\right)$, where $n$ and $m$ are quantum numbers that determine the states of 2D-exciton [17], $n_{e}$ and $n_{h}-$ states of electron and hole that create it. The energy of transition to the exciton state (without account of interaction with phonons) can be represented as the sum

$E_{\mathrm{ex}}^{(0)}=E_{g}+E^{(e)}+E^{(h)}-E_{b}$,

where $E_{g}$ is the width of the forbidden band in well material; $E^{(e)}$ are $E^{(h)}$, respectively, energies of electron and hole in QW; $E_{b}$ is the binding energy, the value of which can be determined using one of versions among variation methods (see [13]).

The Hamiltonians for free phonons and electronphonon interaction in double heterostructures (in coordinate representation by electron variables) were obtained in $[18,19]$. In the case of the system studied here, the former acquires the following look:

$$
\begin{aligned}
& \hat{H}_{\mathrm{ph}}=\hat{H}_{\mathrm{LO}}+\hat{H}_{\mathrm{I}}=\sum_{\lambda, \vec{q}_{\perp}} \Omega_{\mathrm{LO}}\left(\hat{b}_{\lambda \vec{q}_{\perp}} \hat{b}_{\lambda \vec{q}_{\perp}}+1 / 2\right)+ \\
& +\sum_{\sigma=S, A} \sum_{\vec{q}_{\perp}} \Omega_{\sigma}\left(\vec{q}_{\perp}\right)\left(\hat{b}_{\sigma \vec{q}_{\perp}}^{+} \hat{b}_{\sigma \vec{q}_{\perp}}+1 / 2\right),
\end{aligned}
$$

where $\Omega_{\text {LO }}$ is the energy of longitudinal optical (LO) polarization phonons confined in QW medium; $\hat{b}_{\lambda \vec{q}_{\perp}}^{+}$and $\hat{b}_{\lambda \vec{q}_{\perp}}$ are operators of creation and annihilation of the LOphonon state with the wave vector $\vec{q}=\left(q_{\|}, \vec{q}_{\perp}\right)$, the longitudinal component of which $\left(q_{\|}\right)$is quantized and acquires discrete values from the set $\lambda \pi / N c(\lambda=1,2, \ldots$, $N) ; \Omega_{\sigma}\left(\vec{q}_{\perp}\right)$ is the energy of interface (I) phonons for symmetric $(\sigma=\mathrm{S})$ or anti-symmetric $(\sigma=A)$ modes; $\hat{b}_{\sigma \vec{q}_{\perp}}^{+}\left(\hat{b}_{\sigma \vec{q}_{\perp}}\right)$ - operator of creation (annihilation) of the respective I-phonon state.

The Hamiltonian for EPI [18, 19] expressed by the authors of [13] in the secondary quantization representation by all the variables of this electron-phonon system is as follows:

$$
\begin{aligned}
& \hat{H}_{\mathrm{int}}=\hat{H}_{\mathrm{ex}-\mathrm{LO}}+\hat{H}_{\mathrm{ex}-\mathrm{I}}= \\
& =\sum_{p=e, h} \eta_{p}\left\{\sum _ { n _ { p } , n _ { p } ^ { \prime } , \vec { k } _ { \perp } } \left[\sum_{\lambda, \vec{q}_{\perp}} F_{n_{p} n_{p}^{\prime}}^{\lambda}\left(\vec{q}_{\perp}\right) \hat{a}_{n_{p}^{\prime} \vec{k}_{\perp}+\vec{q}_{\perp}} \hat{a}_{n_{p} \vec{k}} \hat{B}_{\lambda \vec{q}_{\perp}}+\right.\right. \\
& \left.\left.\sum_{\sigma, \vec{q}_{\perp}} F_{n_{p} n_{p}^{\prime}}^{\sigma}\left(\vec{q}_{\perp}\right) \hat{a}_{n_{p}^{\prime} \vec{k}_{\perp}+\vec{q}_{\perp}} \hat{a}_{n_{p} \vec{k}_{\perp}} \hat{B}_{\sigma \vec{q}_{\perp}}\right]\right\},
\end{aligned}
$$

and is determined by the functions of electron-phonon coupling $F_{n n^{\prime}}^{\alpha}\left(\vec{q}_{\perp}\right)$, the apparent look of which for QW of the finite depth was adduced in [13], and of the infinite one 
- in [16]. Here, $\eta_{e}=1, \eta_{h}=-1 ; n$ and $n^{\prime}$ - numbers of electron sub-bands in the state $\left(n, \vec{k}_{\perp}\right) ; \alpha$-index of the phonon branch equal to $\lambda$ in the case of LO or $\sigma-$ in the case of I-phonons; $\hat{B}_{\alpha \vec{q}_{\perp}}=\hat{b}_{\alpha \vec{q}_{\perp}}+\hat{b}_{\alpha-\vec{q}_{\perp}}^{+}-$phonon operators, while $\hat{a}_{n \vec{k}_{\perp}}$ and $\hat{a}_{n \vec{k}_{\perp}}^{+}$- electron operators of secondary quantization.

The Hamiltonian (9) was obtained in assumption that EPI is realized via individual interaction between electron and hole, which create exciton, with phonons. This interaction re-normalizes the energy spectrum of quasiparticles causing the shift of their states along the energy scale. In accord with (7), it is pronounced in the change of the exciton transition energy and can be observed as a long-wave shift of the correspondent absorption band. The task of investigations described in this paper was in studying the character of temperature changes in the position and shape of the exciton absorption band in $2 \mathrm{H}$ $\mathrm{PbI}_{2}$ nanofilms embedded into dielectric medium.

\section{Results and discussion}

To create a function describing the shape of the exciton absorption band, the Hamiltonian for EPI can be represented in the following comfortable look [20]:

$\hat{H}_{\text {int }}=\sum_{v, v^{\prime}, \vec{k}_{\perp}} \sum_{\alpha, \vec{q}_{\perp}} \Phi_{v v^{\prime}}^{(\alpha)}\left(\vec{q}_{\perp}\right) \hat{c}_{v^{\prime \prime} \vec{k}_{\perp}+\vec{q}_{\perp}} \hat{c}_{v \vec{k}_{\perp}} \hat{B}_{\alpha, \vec{q}_{\perp}}$,

where $\hat{c}_{v \vec{k}}^{+}$and $\hat{c}_{v \vec{k}}$ are the operators of creation and annihilation for the exciton state in NF $\mid \vec{k}_{\perp}, v>$; $\vec{k}_{\perp}=\vec{k}_{e \perp}-\vec{k}_{h \perp}$ is the transverse component of the exciton wave vector;

$\Phi_{v v^{\prime}}^{(\alpha)}\left(\vec{q}_{\perp}\right)=F_{n_{e^{n_{e}} n^{\prime}}^{\alpha}}^{\alpha}\left(\vec{q}_{\perp}\right) J_{n m, n^{\prime} m^{\prime}}^{(e)}\left(\vec{q}_{\perp}\right)-F_{n_{h} n_{h}^{\prime}}^{\alpha}\left(\vec{q}_{\perp}\right) J_{n m, n^{\prime} m^{\prime}}^{(h)}\left(\vec{q}_{\perp}\right)$

- function of the exciton-phonon coupling;

$I_{n m, n^{\prime} m^{\prime}}^{(p)}\left(\vec{q}_{\perp}\right)=\int \varphi_{n^{\prime} m^{\prime}}^{*}(\vec{\rho}) e^{i\left(\vec{q}_{p}, \vec{\rho}\right)} \varphi_{n m}(\vec{\rho}) d^{2} \rho \quad(p=e, h) ;$

$\vec{q}_{e}=\frac{m_{h \perp}}{m_{\mathrm{ex}}} \vec{q}_{\perp}, \vec{q}_{h}=\frac{m_{e \perp}}{m_{\mathrm{ex}}} \vec{q}_{\perp} ;$

$m_{\mathrm{ex}}=m_{e \perp}+m_{h \perp}-$ effective mass of exciton in the plane of the layered semiconductor layer.

Choosing $\varphi_{n m}(\vec{\rho})$ as a function of the ground ( $n=0, m=0$ ) state for 2D-exciton [17] in the following form

$\varphi_{00}(\vec{\rho})=\sqrt{\frac{8}{\pi a_{\mathrm{ex}}^{2}}} \exp \left(-\frac{2 \rho}{a_{\mathrm{ex}}}\right)$,

the expression (12) can be reduced to the look

$I_{0}^{(p)}\left(\vec{q}_{\perp}\right)=\left[1+\left(q_{p} a_{\mathrm{ex}} / 4\right)^{2}\right]^{-1}$.

Then
$\Phi_{n n^{\prime}}^{(\alpha)}\left(\vec{q}_{\perp}\right)=F_{n n^{\prime}}^{\alpha}\left(\vec{q}_{\perp}\right) \times$
$\times\left[\frac{1}{1+\left(m_{h \perp} a_{\mathrm{ex}} q_{\perp} / 4 m_{\mathrm{ex}}\right)^{2}}-\frac{1}{1+\left(m_{e \perp} a_{\mathrm{ex}} q_{\perp} / 4 m_{\mathrm{ex}}\right)^{2}}\right]$,

where $n=1,2, \ldots$ is the number of the level corresponding to dimensional quantization of carriers in QW.

Using (16), let us represent MO for exciton-phonon interaction (in the case of the ground exciton state) in the form

$$
\begin{aligned}
& M\left(\vec{k}_{\perp}, \omega, T\right)=\sum_{n, \alpha, \vec{q}_{\perp}} \mid \Phi_{1 n}^{(\alpha)}\left(\vec{q}_{\perp}\right)^{2} \times \\
& {\left[\frac{1+v_{\alpha}\left(\vec{q}_{\perp}, T\right)}{\hbar \omega-E_{\mathrm{ex}}\left(\vec{k}_{\perp}-\vec{q}_{\perp}\right)-\Omega_{\alpha}\left(\vec{q}_{\perp}\right)}+\frac{v_{\alpha}\left(\vec{q}_{\perp}, T\right)}{\hbar \omega-E_{\mathrm{ex}}\left(\vec{k}_{\perp}+\vec{q}_{\perp}\right)+\Omega_{\alpha}\left(\vec{q}_{\perp}\right)}\right],}
\end{aligned}
$$

where $\hbar \omega$ is the energy of the incident wave, and $E_{\text {ex }}\left(\vec{k}_{\perp}\right)$ - energy of exciton from the ground exciton band.

In NF of the studied type with the thickness exceeding four layers of lead iodide, interaction with confined phonons is dominant [14]. In this case, with account of the quadratic dispersion law and changing summation by the variable $\vec{q}_{\perp}$ in (17) by integration, and separating real and imaginary parts in it, one can obtain respective analytical expression. The real part of MO

$$
\begin{aligned}
& \Delta(w, T)=-\frac{4 \pi^{2} e^{2}}{a \varepsilon_{0 \perp}^{(0)}} \sum_{n} \sum_{\lambda=1}^{N}\left(\lambda X_{1 n}^{\lambda}\right)^{2} \times \\
& \times\left\{[1+v(T)]\left[J_{\lambda}^{(e,+)}(w, a)+J_{\lambda}^{(h,+)}(w, a)-2 J_{\lambda}^{(e h,+)}(w, a)\right]+\right. \\
& \left.+v(T)\left[J_{\lambda}^{(e,-)}(w, a)+J_{\lambda}^{(h,-)}(w, a)-2 J_{\lambda}^{(e h,-)}(w, a)\right]\right\}
\end{aligned}
$$

where $\varepsilon_{0 \perp}^{(0)}$ is the static dielectric permittivity in the plane of layer package for layered semiconductor (superscript here indicates medium); $X_{1 n}^{\lambda}$ - quantity adduced in [14] that defines interaction of electron (in its ground state in QW $\left.\left(n_{e}=1\right)\right)$ with confined phonon being in the state with a quantum number $\lambda$ with participation of $n$-th $\left(n_{e}=n\right)$ minilevel of carrier; $v(T)$ are the numbers of filling the phonon states:

$$
\begin{aligned}
& J_{\lambda}^{(p, \pm)}(w, a)=\frac{\ln \left|1-\frac{1}{w \mp 1}\right|}{D^{(p, \pm)}(w) G_{\lambda}^{( \pm)}(w, a)}- \\
& -\frac{\ln \left[1+\left(\frac{a}{\lambda a_{0}}\right)^{2}\right]}{\left[A_{\lambda}^{(p)}(a)\right]^{2} G_{\lambda}^{( \pm)}(w, a)}+\frac{\alpha_{p}}{\left(1+\alpha_{p}\right) A_{\lambda}^{(p)}(a) D^{(p, \pm)}(w)}+
\end{aligned}
$$


$+\frac{\alpha_{p} \ln \left(1+\alpha_{p}\right)}{A_{\lambda}^{(p)}(a) D^{(p, \pm)}(w)}\left[\frac{1}{A_{\lambda}^{(p)}(a)}+\frac{1}{D^{(p, \pm)}(w)}\right]$

$J_{\lambda}^{(e h, \pm)}(w, a)=-\frac{1}{A_{\lambda}^{(p)}(a)} \times$

$\times\left[\frac{\alpha_{e}^{2} \ln \left(1+\alpha_{e}\right)}{\left(\alpha_{h}-\alpha_{e}\right) D^{(e, \pm)}(w)}+\frac{\ln \left[1+\left(\frac{a}{\lambda a_{0}}\right)\right]}{A_{\lambda}^{(h)}(a) G_{\lambda}^{( \pm)}(w, a)}\right]+$

$+\frac{1}{D^{(h, \pm)}}\left[\frac{\ln \left|1-\frac{1}{w \mp 1}\right|}{D^{(p, \pm)}(w) G_{\lambda}^{( \pm)}(w, a)}+\frac{\alpha_{h}^{2} \ln \left(1+\alpha_{h}\right)}{A_{\lambda}^{(h)}(a)\left(\alpha_{h}-\alpha_{e}\right)}\right]$.

Here, $a_{0}$ is the lattice parameter in the plane of the layer package,

$A_{\lambda}^{(p)}(a)=1-\alpha_{p}\left(\frac{\lambda a_{0}}{a}\right)^{2}, D^{(p, \pm)}(w)=1+\alpha_{p}(w \mp 1)$,

$G_{\lambda}^{( \pm)}(w, a)=\left(\frac{\lambda a_{0}}{a}\right)^{2}+w \mp 1$

- auxiliary functions, $\alpha_{p}=\left(\frac{\pi m_{p \perp} a_{\mathrm{ex}}}{4 m_{\mathrm{ex}} a_{0}}\right)^{2}, p=(e, h)-$ index of carriers, and $w=\frac{\hbar \omega-E_{\mathrm{ex}}^{(0)}}{\Omega_{\mathrm{LO}}}-$ shift of the maximum inherent to the band of exciton absorption relatively to its position $E_{\mathrm{ex}}^{(0)}=E_{\mathrm{ex}}(0)$. This shift is normalized by the energy of confined phonon and $E_{\mathrm{ex}}(0)$ calculated without taking into account the EPI.

The imaginary part is reduced to the look

$\Gamma(w, T)=\Gamma_{0}+\frac{4 \pi^{3} e^{2}}{a \varepsilon_{0 \perp}^{(0)}} \sum_{n} \sum_{\lambda=1}^{N}\left(\lambda X_{1 n}^{\lambda}\right)^{2} \times$

$\times\left\{[1+v(T)] \frac{\left[I_{0}^{(e)}\left(q_{-}(w)\right)-I_{0}^{(h)}\left(q_{-}(w)\right)\right]^{2}}{\left(a_{0} q_{-}(w) / \pi\right)^{2}+\left(\lambda a_{0} / a\right)^{2}}+\right.$

$\left.+\frac{\left[I_{0}^{(e)}\left(q_{+}(w)\right)-I_{0}^{(h)}\left(q_{+}(w)\right)\right]^{2}}{\left(a_{0} q_{+}(w) / \pi\right)^{2}+\left(\lambda a_{0} / a\right)^{2}} v(T)\right\}$,

where $q_{ \pm}(w)=\pi \sqrt{1 \pm w} / a_{0}$, and $\Gamma_{0}$ is a phenomenologic constant that takes into account exciton relaxation via processes not accounted in this model.

Using $2 \mathrm{H}-\mathrm{PbI}_{2}$ parameters adduced in [14] and those of ambient medium as well as experimentally determined value $\Gamma_{0}=10 \mathrm{meV}$, the authors calculated spectral dependences for the normalized function describing the shape of exciton absorption band in NF of various thickness at $T=0 \mathrm{~K}$ with account of spatial confinement, self-polarization of NF surfaces and EPI. The results of these calculations showed that interaction with virtual phonons shifts the peak of exciton absorption band to the long-wave spectral range, and the larger is the NF thickness, the stronger is this shift. At the NF thickness equal to $4 \ldots 7$ layers of $2 \mathrm{H}-\mathrm{PbI}_{2}$ in $\mathrm{E}-$ MAA matrix, the shift value is $22 \ldots 35 \mathrm{meV}$, while that in glass $-18 \ldots 35 \mathrm{meV}$. With increasing the NF thickness, the shift magnitude approaches to the value typical to bulk $2 \mathrm{H}-\mathrm{PbI}_{2}$ crystal (i.e., $40 \mathrm{meV}$ ). The band width remains practically unchanged, since at $T=0$ processes of absorption and emission of real phonons that cause widening are absent.

The character of temperature changes observed in the shape of absorption band, which is calculated for the case of $\mathrm{NF} \mathrm{PbI}_{2} / \mathrm{E}-\mathrm{MAA}$ with the thickness of 7 layers from lead iodide and with account of interaction exclusively with LO-phonons, shows that essential temperature changes in the band position and shape begin at $T \geq 75 \mathrm{~K}$. The increase in temperature causes a monotonic shift of the exciton peak to the long-wave range, and at $300 \mathrm{~K}$ this shift reaches $80 \mathrm{meV}$ (Fig. 1), which is in good accordance with the data of experimental measurements [4]. In this case, the band width grows insignificantly as a consequence of exciton scattering by LO-phonons (Fig. 2). The height of the peak is respectively lowered, which is in good accordance with the implication of the theory developed for exciton absorption in the case of optical phonon participation - changes in temperature do not change the area of the band [21].

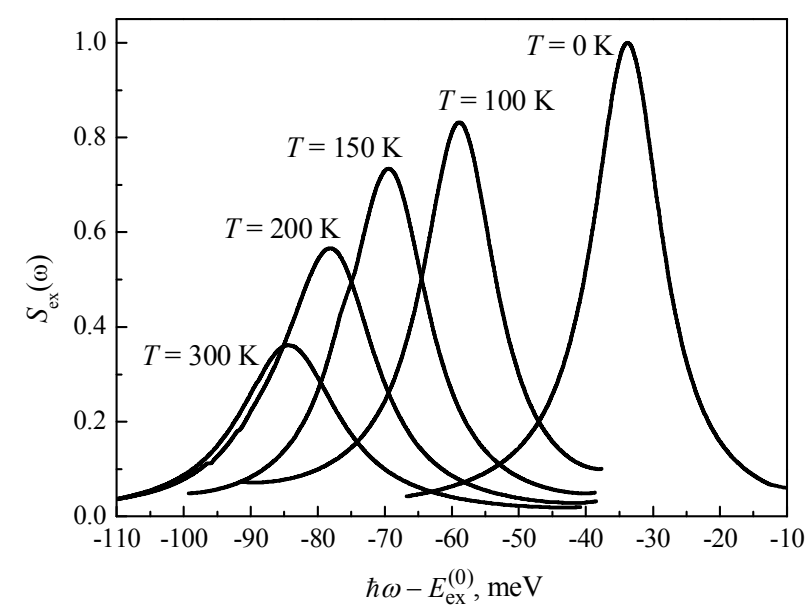

Fig. 1. Temperature changes in the shape of exciton absorption band. 


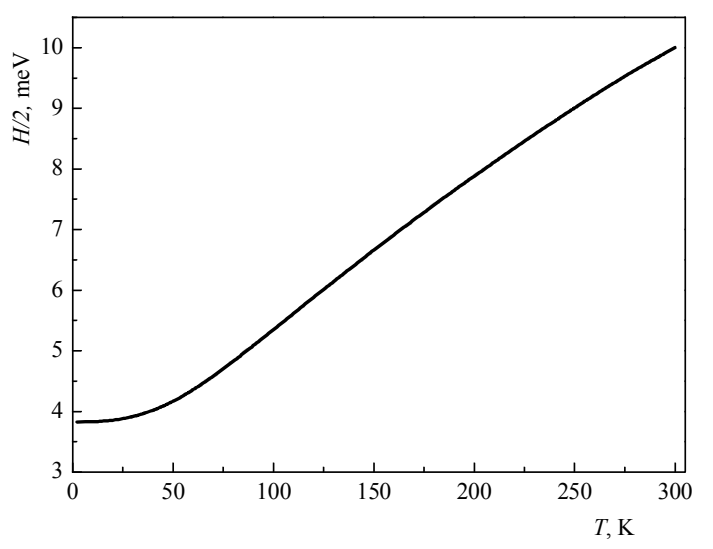

Fig. 2. Temperature changes in the halfwidth of exciton absorption band.

\section{Conclusions}

Obtained in this work are the expressions for calculations of temperature dependences of the shape inherent to the band of exciton absorption in plain nanoheterostructures with a single quantum well. Calculations performed using as an example the nanofilm $2 \mathrm{H}-\mathrm{PbI}_{2}$ embedded into glass or polymer E-MAA matrix have shown that essential temperature changes in the position and shape of the absorption band begin at $T \geq 75 \mathrm{~K}$. Growth of the NF temperature causes a monotonic shift of the exciton peak to the long-wave spectral range, and this shift reaches $80 \mathrm{meV}$ at $300 \mathrm{~K}$. In this case, the width of the band is increased to some extent and becomes $4 \ldots 10 \mathrm{meV}$. The results of these calculations are in good accordance with the data of experimental measurements [3-5].

\section{References}

1. L. Fornaro, E. Saucedo, L. Mussio, A. Gancharov, Growth of lead iodide platelets for room temperature X-ray detection by the vapor transport method // Proc. SPIE, 4507, p. 90-98 (2001).

2. C.E. Finlayson and P.J.A. Sazio, Highly efficient blue photoluminescence from colloidal lead-iodide nanoparticles // J. Phys. D: Appl. Phys. 39(8), p. $1477-1480$ (2006).

3. I.Kh. Akopyan, O.N. Volkova, B.V. Novikov, B.I. Venzel, Size effects in the optical spectra of the microcrystals $\mathrm{PbI}_{2}$ and $\mathrm{HgI}_{2}$ // Fizika tverdogo tela, 39(3), p. 468-473 (1997), in Russian.

4. A.S. Ablitzova, V.F. Aguekian, A.Yu. Serov, Optical spectra of microcrystals of a layer semiconductor $\mathrm{PbI}_{2}$ in porous glass matrices // Fizika i tekhnika poluprov. 32(2), p. 151-154 (1998), in Russian.

5. A. Yamamoto, H. Nakahara, S. Yano, T. Goto, and A. Kasuya, Exciton dynamics in $\mathrm{PbI}_{2}$ ultra-thin microcrystallites // Phys. status solidi (b) 224(1), p. 301-305 (2001).

6. A.I. Savchuk, V.I. Fediv, Ye.O. Kandyba, T.A. Savchuk, I.D. Stolyarchuk, P.I. Nikitin,
Platelet-shapped nanoparticles of $\mathrm{PbI}_{2}$ and $\mathrm{PbMnI}_{2}$ embedded in polimer matrix // Mat. Sci. Eng. C, 19(1-2), p. 59-62 (2002).

7. N. Preda, L. Mihut, I. Baltog, T. Velula, V. Teodorescu, Optical properties of lowdimensional $\mathrm{PbI}_{2}$ particles embedded in polyacrylamide matrix // J. Optoelectron. \& Adv. Materials, 8(3), p. 909-913 (2006).

8. N. Preda, L. Mihut, M. Baibarac, I. Baltog, Raman and photoluminescence studies on low-dimensional $\mathrm{PbI}_{2}$ particles embedded in polymer matrix // J. Optoelectron. \& Adv. Materials, 9(5), p. 1358-1361 (2007).

9. I. Saikumar, Sh. Ahmad, J.J. Baumberg, G. Vijaya Prakash, Fabrication of excitonic luminescent inorganic-organic hybrid nano- and microcrystals // Scr. Mater. 67, p. 834-837 (2012).

10. R. Zheng, M. Matsuura, T. Taguchi, Exciton-LOphonon interaction in zinc-compound quantum wells // Phys. Rev. B, 61(15), p. 9960-9963 (2000).

11. R.T. Senger, K.K. Bajaj, Binding energy of excitons in II-VI compound-semiconductor based quantum well structures // Phys. status solidi (b), 241(8), p. 1896-1900 (2004).

12. I.V. Ponomarev, L.I. Deych, V.A. Shuvayev, A.A. Lisyansky, Self-consistent approach for calculations of exciton binding energy in quantum wells // Physica E, 25, p. 539-553 (2005).

13. V.M. Kramar, M.V. Tkach, Exciton-phonon interaction and exciton energy in semiconductor nanofilms // Ukr. J. Phys. 54(10), p. 1027-1035 (2009).

14. O.V. Pugantseva, V.M. Kramar, I.V. Fesiv, O.O. Kudryavtsev, Temperature changes of the exciton transition energy in lead di-iodide nanofilms // Semiconductor Physics, Quantum Electronics and Optoelectronics, 16(2), p. 170-176 (2013).

15. M.V. Tkach, Quasi-particles in Nanoheterosystems. Quantum Dots and Wires: A Manual. Chernivtsi Univ. Press, Chernivtsi, 2003 (in Ukrainian).

16. O.V. Pugantseva, V.M. Kramar, Self-polarization effect and electron-phonon interaction contributions in forming of electron energy spectrum of $\mathrm{PbI}_{2}$ nanofilm embed in E-MAA copolymer // J. NanoElectron. Phys. 4(4), 04021(6 pp.) (2012).

17. M. Shinada, S. Sugano, Interband optical transitions in extremly anisotropic semiconductors // J. Phys. Soc. Jpn. 21(10), p. 1936-1946 (1966).

18. L. Wendler, R. Pechstedt, Dynamical screening, collective excitations, and electron-phonon interaction in heterostructures and semiconductor quantum wells // Phys. status solidi (b) 141(1), p. 129-150 (1987).

19. N. Mori, T. Ando, Electron-optical-phonon interaction in single and double heterostructures // Phys. Rev. B, 40(9), p. 6175-6188 (1989).

20. A.S. Davydov, Theory of Solids. Nauka, Moscow, 1976 (in Russian).

21. M.S. Brodin, I.V. Blonskii, Excitonic Processes in Layered Crystals. Naukova Dumka, Kiev, 1986 (in Russian). 